\title{
Hydrodynamics of droplet impingement on hot surfaces of varying wettability
}

\author{
Cristian E. Clavijo, Julie Crockett, Daniel Maynes \\ Mechanical Engineering, BYU, Provo, UT 84602
}

\begin{abstract}
This work presents on the hydrodynamics of water droplet impingement on superheated solid surfaces across the entire wettability spectrum: superhydrophilic, hydrophilic, hydrophobic and superhydrophobic. While a large body of work exists on droplet impingement on hydrophilic and superhydrophilic surfaces, impingement on the latter two has been largely neglected and the present results show that dynamics are dramatically different. Experiments ranging in surface temperature from $125^{\circ} \mathrm{C}$ to $415^{\circ} \mathrm{C}$ and Weber numbers from 10 to 225 were performed and analyzed using high-speed imaging. Some of the most striking differences are as follows. While atomization is always present for impingement on the hydrophilic and superhydrophilic surfaces at temperatures below the Leidenfrost point, atomization is absent at low Weber numbers and at low excess surface temperatures on the hydrophobic surface. At high surface temperatures, the attraction of vapor bubbles on the hydrophobic surface allows a vapor blanket to form more readily thus leading to Leidenfrost behavior at a much lower temperature than classically observed on a hydrophilic surface. One of the most interesting phenomenon that will be discussed includes what will be described as a "pseudo-Leidenfrost" state for impingement on the superhydrophobic surface. Because water can be suspended at the peaks of the roughness on a superhydrophobic interface, vapor escapes from underneath the droplet thus mimicking Leidenfrost behavior for all excess temperatures. This results in minimal atomization for superhydrophobic impingement over the entire regime explored. Finally, maximum spread diameters for Leidenfrost impingement are tabulated as a function of the Weber number for all surfaces and are shown to be larger on the smooth surfaces than on the textured ones indicating that droplet spreading at the Leidenfrost point is not independent
\end{abstract}

Preprint submitted to International Journal of Heat and Mass Transfer August 24, 2016

(C) 2016. This manuscript version is made available under the Elsevier user license

http://www.elsevier.com/open-access/userlicense/1.0/ 
of surface type as previously supposed.

Keywords: superhydrophobic, droplet impingement, boiling

\section{Introduction}

Significant interest in the interaction between droplets and superheated solid surfaces is evident by the plethora of publications in the last few years $[1,2,3,4,5,6,7,8,9,10]$. Droplet impingement is present in a wide array of applications including spray cooling, coating, biochemical reactions and combustion, and is the focus of this paper. The physics involved are rich and complex. From a hydrodynamical standpoint, a droplet impinging a hot surface may boil violently, atomize and splash, or rebound without either splashing or atomizing (or a combination of the two). Instantaneous heat transfer rates correspondingly vary dramatically across these different regimes. While ample research has been performed in this field, most of it has focused on high energy surfaces relative to the liquid [10, 11, 12, 13]. In this work, we explore single droplet impingement on hydrophobic and superhydrophobic surfaces heated above the saturation temperature of the liquid.

Boiling regime maps for water droplet impingement on heated smooth hydrophilic surfaces show contact boiling increases with increasing surface temperature at low excess temperatures [11]. When boiling, a droplet becomes populated with vapor bubbles that can burst and eject tiny droplets into the surrounding gas. The ejection of these tiny droplets is generally referred to as atomization or secondary atomization; we will refer to it as atomization. It is now well established that for relatively high surface temperatures, atomization ceases to occur and the droplet rebounds as if on a superhydrophobic surface, despite the strong liquid-solid attraction. The lack of atomization is attributed to the presence of a thin vapor film underneath the droplet through which vapor can escape, thus preventing vapor bubbles from forming within the droplet $[10,11,12,13]$ and this regime is known as film boiling. The lowest surface temperature at which atomization ceases to occur has been named the Leidenfrost point (LFP) [14, 15, 16, 17]. Tran et al. analyzed film boiling dynamics using an optically-transparent sapphire plate as the impinged surface and observed that the liquid does not contact the solid for impingement during film boiling [12], supporting the theory of a thin vapor film below the droplet and resultant lack of atomization. 
A balance between vapor production, associated with the surface temperature, and inertia of the impacting droplet, associated with the Weber number $W e=\rho V^{2} D / \sigma$ (where $\rho, \sigma, V$ and $D$ represent liquid density and surface tension, impact velocity and initial diameter, respectively), defines the transition between contact and film boiling. Consequently, this transition temperature (i.e. the LFP) increases with increasing Weber number $[11,10,12,13]$. As surface temperature increases, well above the LFP, an entirely new regime termed "spray film boiling" has been observed on smooth hydrophilic surfaces [12]. We limit the scope of this work to surface temperatures below this regime and therefore no atomization was observed at temperatures above the LFP.

Texturing hydrophilic surfaces has been shown to alter vapor generation and vapor flow dynamics below the droplet. Micropillars can decrease the LFP temperature at a given $W e$ due to enhanced vapor production $[13,18]$. To test this hypothesis, Tran et al. showed that as pillar height increases the LFP decreases, supporting the idea that enhanced solid surface area (by texturing) during impingement promotes the formation of the vapor film at a lower temperature [13]. Interestingly, it seems that the very densely packed micropillars can have an opposite effect. Zhang et al. found that the LFP increased for such textured surfaces due to suppressed lateral vapor escape [18], which has also been reported elsewhere [5, 19].

The maximum spread diameter, $D_{\max }$, at the LFP provides insight to the dynamics of the system as well and has been explored in multiple previous works $[9,20,21,22,23]$. Tran et al. showed that $D_{\max }$ is larger at the LFP on a hydrophilic surface than on a superhydrophobic surface maintained at room temperature for $W e<1000$, although both exhibit similar spreading/retracting dynamics [12]. These trends have also been confirmed elsewhere [24]. Larger maximum spread diameters during Leidenfrost impingement are attributed to droplets being hotter (less viscous) and not interacting with the solid (less frictional resistance). These results seem to indicate that droplet spreading/retracting dynamics in the film boiling regime may be independent of the wettability of the surface since the droplet is suspended above its own vapor with negligible contact with the solid [13].

Though previous research has covered a broad range of experimental conditions and provided great insight into the physics of the interaction between a droplet and a heated hydrophilic surface, impingement on hydrophobic and superhydrophobic surfaces at elevated temperatures has received only modest attention $[9,25,26,18]$. Park et al. performed experiments of droplets 
(360 $\mu \mathrm{m}$ in diameter) impinging at $W e=60$ on hydrophilic and hydrophobic surfaces over a temperature range of $110^{\circ} \mathrm{C}$ to $210^{\circ} \mathrm{C}$ [26]. However, because the study was mainly focused on thermal transport considerations between solid and droplet, little information on boiling hydrodynamics was provided. They reported that the residence time was generally lower for impingement on a hydrophobic substrate, but no information regarding Leidenfrost transition temperatures or maximum spread diameter at the LFP was given. In a different work, $\mathrm{Li}$ et al. reported hydrodynamic behavior of impinging droplets at $W e=22$, such as droplet height, diameter and dynamic contact angles, on surfaces of varying wettability (hydrophilic to superhydrophobic) but the maximum surface temperature explored was limited to $110^{\circ} \mathrm{C}[9]$. More recently, Zhang et al. explored impingement on superhydrophobic surfaces with microscale posts in a square lattice [18] for Weber numbers up to 85 and temperatures up to $320^{\circ} \mathrm{C}$. They mapped different behaviors including contact boiling and rebound with and without satellite droplets (at around the LFP). The LFP temperature is lower for droplet impingement on the sparsely spaced micropillars than on the more densely packed micropillars. This can be explained again by a balance of vapor generation and micropillars blocking the vapor flow, which is similar to what occurs on hydrophilic surfaces [18]. For sessile droplets the boiling dynamics on heated superhydrophobic surfaces $\left(<230^{\circ} \mathrm{C}\right)$ is minimal relative to hydrophobic surfaces [27]. Additionally, a shift to higher Leidenfrost temperatures for superhydrophobic surfaces relative to smooth hydrophobic $[27,28]$ and superhydrophilic relative to smooth hydrophilic surfaces [29, 30] has been observed, but only for static droplet scenarios. Thus, hydrodynamics of impinging droplets on hydrophobic and superhydrophobic surfaces at temperatures well above saturation remains largely unexplored.

This paper presents on the phenomena of a millimetric water droplet impinging with Weber numbers from 10 to 225 on heated surfaces across the entire wettability spectrum (superhydrophilic to superhydrophobic). The range of surface temperatures explored is $125^{\circ} \mathrm{C}$ to $415^{\circ} \mathrm{C}$. Given that a broad body of work on hydrophilic/superhydrophilic impingement already exists, the main contribution of this work is attributed to dynamics on the hydrophobic and superhydrophobic surfaces. Notwithstanding, experiments were also conducted on hydrophilic and superhydrophilic surfaces for comparison with other works. Section 2 provides a detailed methodology of the experimental approach. In Section 3, high-speed images are provided which qualitatively show the difference in atomization and vapor bubble formation across the 
different types of surfaces for varying $W e$ and surface temperatures. Atomization regime maps are constructed for each surface, which clearly identify the LFP as a function of the Weber number. Maximum spread diameters at the LFP as a function of time and impact Weber number are also provided. Brief conclusions are given in Section 4.

\section{Experimental procedure}

Four surface types were fabricated on $500 \mu \mathrm{m}$ thick / $100 \mathrm{~mm}$ diameter polished silicon wafers: superhydrophilic (SHL), hydrophilic (HL), hydrophobic (HB) and superhydrophobic (SHB). For the HL surface, an unaltered polished silicon wafer was used yielding a static contact angle of $30^{\circ} \pm 3^{\circ}$. For the HB surface, a $200 \mathrm{~nm}$ coat of Teflon was applied to a wafer in the following manner to render it hydrophobic. Teflon (4, 5-difluoro2,2-bis(trifluoromethyl)-1,3-dioxole) was dissolved in FC-40 to yield a $0.2 \%$ Teflon solution, which was subsequently applied to the wafer by spin coating ( $\mathrm{a} \sim 100 \mathrm{~nm}$ chromium layer was applied first for adhesion promotion). The wafer was then placed on a hot plate at $90^{\circ} \mathrm{C}$ for 5 minutes, $165^{\circ} \mathrm{C}$ for 5 minutes and $330^{\circ} \mathrm{C}$ for 20 minutes. Teflon was chosen as the coating material due to its stronger thermal stability than other hydrophobic coats such as organosilanes. The receding and advancing contact angle on the HB surface were $113^{\circ}$ and $128^{\circ} \pm 3^{\circ}$, respectively. For the SHL and SHB surfaces, wafers were first arrayed with a square lattice arrangement of micro-pillars via photolithography and etching (6 $\mu \mathrm{m}$ diameter, $8 \mu \mathrm{m}$ height and $16 \mu \mathrm{m}$ center-to-center spacing). Teflon was applied in a similar manner to the description above to yield the SHB surface, while the SHL surface was left uncoated. Roughness is known to increase hydrophilicity/phobicity on a given surface per the Wenzel and Cassie-Baxter equations [31, 32], respectively: $\cos \theta=r \cos \theta_{e}$ and $\cos \theta=\phi \cos \theta_{e}+\phi-1$, where $\theta, r, \theta_{e}$ and $\phi$ respectively represent the apparent contact angle, the overall surface area divided by its projected area (roughness), intrinsic contact angle, and the area of the top of the pillars divided by the projected area of the surface (solid fraction). For the SHB and SHL surfaces $r \approx 1.6$ and $\phi \approx 0.11$. The receding and advancing contact angles on the SHB surface were $145^{\circ}$ and $165^{\circ} \pm 3^{\circ}$, respectively, while the same angles for the SHL surface were both negligible. SEM images and sessile water droplets on all four surfaces are shown in Figure 1.

For heating, an aluminum block was embedded with four $500 \mathrm{~W}$ cartridge heaters upon which the wafers were placed. The surface temperature of the 


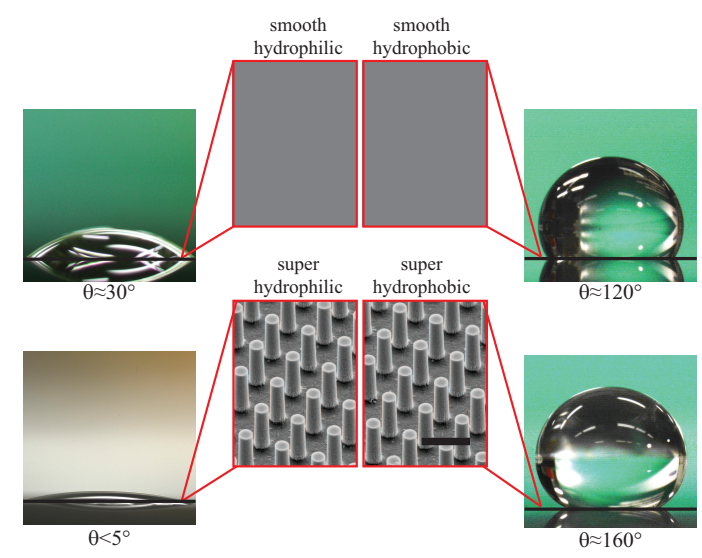

Figure 1: A $5 \mu \mathrm{L}$ water droplet resting on the four different surfaces used in this work: (top) hydrophilic and hydrophobic; (bottom) superhydrophilic and superhydrophobic.

wafer, $T_{s}$, (the value reported in this work as "surface temperature before impact") was deduced with four thermocouples placed $5 \mathrm{~mm}$ below the top of the aluminum block. They were calibrated by placing a silicon wafer coated with a thin coat of known emissivity (0.96) and measuring its temperature with a FLIR SC103 high-speed IR thermal imaging camera. This was accomplished for a smooth wafer, a Teflon-coated smooth wafer (with the same coating thickness as the HB surface) and a micro-pillar structured wafer. All surfaces yielded an equal differential reading between thermal camera and thermocouples. Thermal camera values were usually $2^{\circ} \mathrm{C}-10^{\circ} \mathrm{C}$ lower than thermocouple readings depending on the temperature of the block. Figure 2 shows the relation between the thermal camera measurements and thermocouple measurements (symbols) and a linear fit up to $350^{\circ} \mathrm{C}$, a limit imposed by the coat of known emissivity. For block temperatures above this point, the surface temperature of the wafer was deduced by extrapolation. The range of surface temperatures tested in this work was $125^{\circ} \mathrm{C}-415^{\circ} \mathrm{C}$ for the $\mathrm{HL}$ and SHL surfaces, and $125^{\circ} \mathrm{C}-340^{\circ} \mathrm{C}$ on the HB and SHB surfaces. The temperature range on the hydrophobic surfaces was limited by the glass transition temperature of Teflon. Water contact angle tests were performed on the HB and SHB surfaces regularly after experiments to verify that no Teflon degradation had occurred.

Impingement events were captured in the following manner. A distilled water droplet ( $5 \mu \mathrm{L}, D=2.2 \mathrm{~mm}$ was constant across all experiments) was released from a syringe (needle gauge 30 ) fixed some height above the heated 


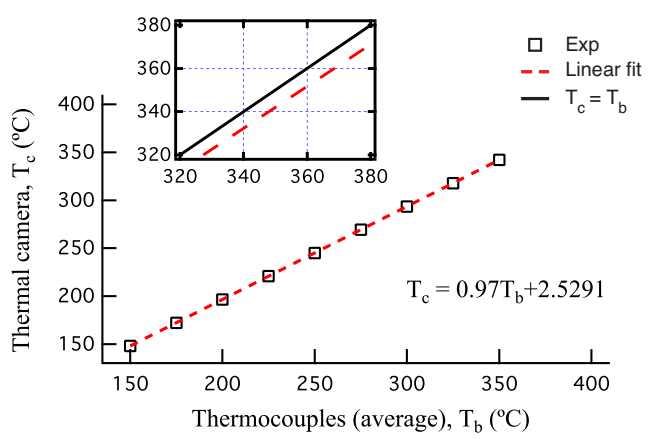

Figure 2: Calibration plot between temperature measured by thermocouples and thermal camera.

wall such that the impact Weber number was varied by adjusting the release height. All events were captured with an APX RS Photron (Model: MONO) high-speed camera at 3000 - 6000 fps with a spatial resolution of $12 \mu \mathrm{m} /$ pixel. Regime maps were constructed based on whether atomization was observed. Atomization was defined as a mist of very small droplets that are ejected during spreading and did not include satellite droplet separation at the periphery of the drop. The LFP was defined as the lowest surface temperature at which atomization was no longer observed. Whether atomization was present in a given scenario was determined by visual inspection of the high-speed videos. While the presence of atomization was generally easy to detect, determining its presence near transitional boundaries (onset of film boiling) was not straightforward in some cases. The reason for this is that atomization decays as surface temperature nears the LFP such that its intensity slowly decreases making the definite temperature at which it completely stops difficult to establish. Furthermore, it has been said that atomization dynamics near the LFP are probabilistic [10]. This is why the transition from the atomization to atomization-free regimes is generally given with a temperature resolution of $10^{\circ} \mathrm{C}$ to $40^{\circ} \mathrm{C}[10,12,13]$. For the present work, the resolution is $25^{\circ} \mathrm{C}$.

Impact parameters such as droplet velocity and initial and maximum spreading diameters were extracted via a computer vision algorithm. Impact droplet diameter was obtained by $D=\sqrt{4 A / \pi}$, where $A$ was the area captured by the camera and the impact velocity was calculated by fitting a straight line through the center of gravity of the descending droplet during the last 15 - 20 frames prior to impact. Liquid properties such as density and 
surface tension were always evaluated at room temperature. Uncertainty in the droplet diameter and the impact Weber number is about $1 \%$ and $3 \%$, respectively. Each scenario was repeated between 5 - 12 times and the total uncertainty was based on a T-test $95 \%$ confidence interval.

\section{Results \& Discussion}

Results are organized as follows. In Section 3.1, high-speed images of droplet impingement on all four surface types are shown for different Weber numbers and surface temperatures. Hypotheses are postulated based on the observations and discussed qualitatively. In Section 3.2, atomization regime maps are presented. LFP values are compared with other work available in the literature and differences across varying surface types are addressed. In Section 3.3, maximum spread diameters as a function of $W e$ for all surfaces at their respective LFP are presented. We note that this study is only concerned with the initial rebound of a droplet (typically within $12 \mathrm{~ms}$ of impact) and not subsequent ones.

\subsection{High Speed Imaging of Atomization}

Basic differences between the SHL/HL and the SHB/HB surfaces at low $T_{s}$ and low $W e$ will be discussed first. This scenario is depicted through the high-speed image sequence in Fig. 3, where the rows (from top to bottom) represent impingement on SHL, HL, HB and SHB surfaces, respectively, at $T_{s}=150^{\circ} \mathrm{C}$ and $W e=40$. The times for all frames are given in the figure, where maximum spread diameter on the $\mathrm{HB}$ surface occurs at $\approx 3 \mathrm{~ms}$. The photographs show that atomization does not begin on any of the surfaces by $3 \mathrm{~ms}$. At $9 \mathrm{~ms}$, the droplets on the SHL and HL surfaces have completely spread out over the surface and exhibit atomization, while the droplets on the HB and SHB surfaces are rebounding with no atomization having taken place. Atomization on the SHL surface is more abundant than on the HL surface because of the increase in surface area due to the texturing, having also been reasoned elsewhere [13]. The spread-and-stick behavior on the HL and SHL surfaces, as well as the repelling behavior on the HB and SHB ones, is similar to impingement at room temperature given the low excess temperature here. Because the liquid remains attached on the former two surfaces, it will eventually completely vaporize for large enough times $(\sim 1$ s). On the other hand, the lack of atomization on the HB and SHB surfaces may be attributed to the smaller maximum spread diameter (smaller contact 


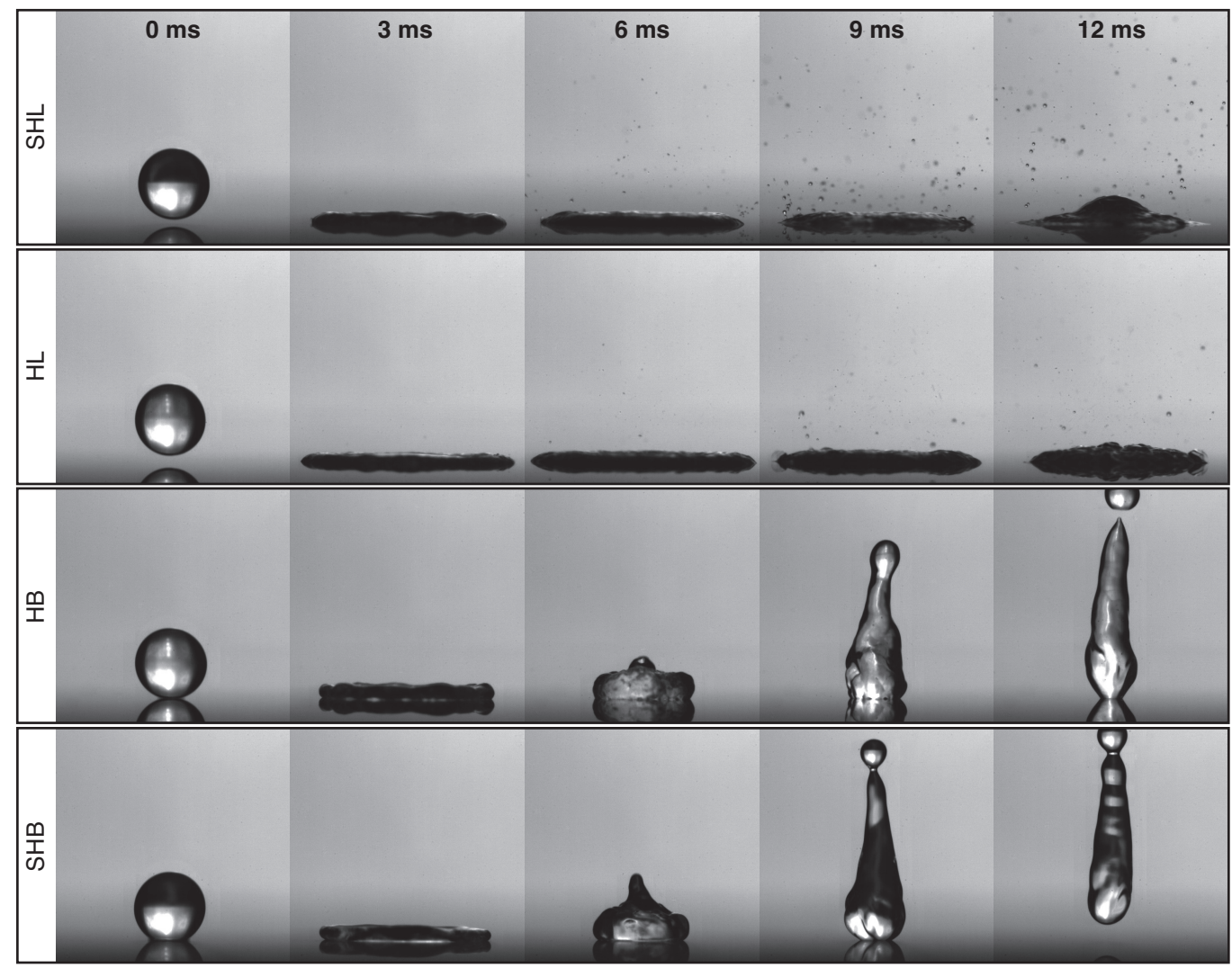

Figure 3: Image sequences of droplet impingement on all four surfaces at $T_{s}=150^{\circ} \mathrm{C}$ and $W e=40$. Atomization exists on the SHL and HL surfaces and is absent on the HB and SHB surfaces.

area) and less time spent in contact with the surface, both of which result in less heat transfer. Given that atomization is a consequence of bursting bubbles, the presence of atomization on the HL and SHL surfaces implies the presence of bubbles on these surfaces. This is not surprising since the surface temperature was $50^{\circ} \mathrm{C}$ above saturation temperature prior to impact. However, it is interesting that no atomization results on the $\mathrm{HB}$ and $\mathrm{SHB}$ surfaces since we would expect bubble formation on these surfaces as well due to surface temperature. Therefore, if bubbles exist on the HB and SHB surfaces, why do they not burst?

To determine the presence of bubbles on the HB and SHB surfaces, impingement was filmed from a top view at the same surface temperature and Weber number. The photographs are shown in Fig. 4 and depict dynamics 
which occur before the rebounding jet begins to form on the HB and SHB surfaces $(\approx 4.3 \mathrm{~ms})$. As seen from this view, it is evident that the spread diameter is generally larger on the HL and SHL surfaces as already discussed. Furthermore, the presence of vapor bubbles is verified here on these surfaces, as well as on the HB and SHB surf aces. The presence of vapor bubbles but lack of atomization (at least any that could be captured by the same camera arrangement that captured atomization on the other surfaces) then suggests that no bubble bursting occurs. One possible explanation is that while the liquid lamellar film on the HL and SHL surfaces is sufficiently thin as to allow "venting of the vapor bubbles" through bursting and atomization [12], the film remains thicker than the bubbles on the HB and SHB surfaces hence no atomization results. The larger spread diameters on the HL and SHL surfaces support the idea of a thinner liquid lamella during impingement. This establishes an important technicality often used-or misused-loosely in the field: contact boiling and atomization for droplet impingement should not be used interchangeably since these are due to different mechanisms. Here we see that contact boiling may be present at low excess temperatures while the atomization regime has still not been reached.

Next, we continue to explore dynamics on all surfaces at low We but higher surface temperatures. The top panel of Fig. 5 shows impingement dynamics at $T=275^{\circ} \mathrm{C}$ and $W e=25$. Atomization is evident on the HL and SHL surfaces as expected at this temperature. Similar to the results of Fig. 3, no atomization occurs on either the HB or SHB surface. First, dynamics on the HB surface will be discussed. The lack of atomization here is unexpected since spreading on the HB surface exhibits diameters of similar lengths to the HL surfaces (which was not the case at $W e=40$ ) and thus similar thin film thickness and boiling behavior should prevail. Experiments were performed at the same $W e$ for lower temperatures to test whether the film boiling regime had been reached. These experiments, for which images are not shown, resulted in atomization over the range $200^{\circ} \mathrm{C}<T_{s}<270^{\circ} \mathrm{C}$ indicating that the LFP on the HB surface occurs at $T_{s} \approx 275^{\circ} \mathrm{C}$. This result is intriguing because transition to film boiling at this $W e$ does not occur on the HL and SHL surfaces until $T_{s} \approx 365^{\circ} \mathrm{C}$ and $320^{\circ} \mathrm{C}$, respectively. What causes the LFP to occur on a hydrophobic surface at lower temperatures? It has been shown previously that vapor bubbles on smooth hydrophobic substrates tend to grow in a flat manner and spread out over the solid (analogous to a water droplet on a hydrophilic surface), which is in contrast to bubble growth on a hydrophilic surface where bubbles bead up (analogous to a water droplet on 


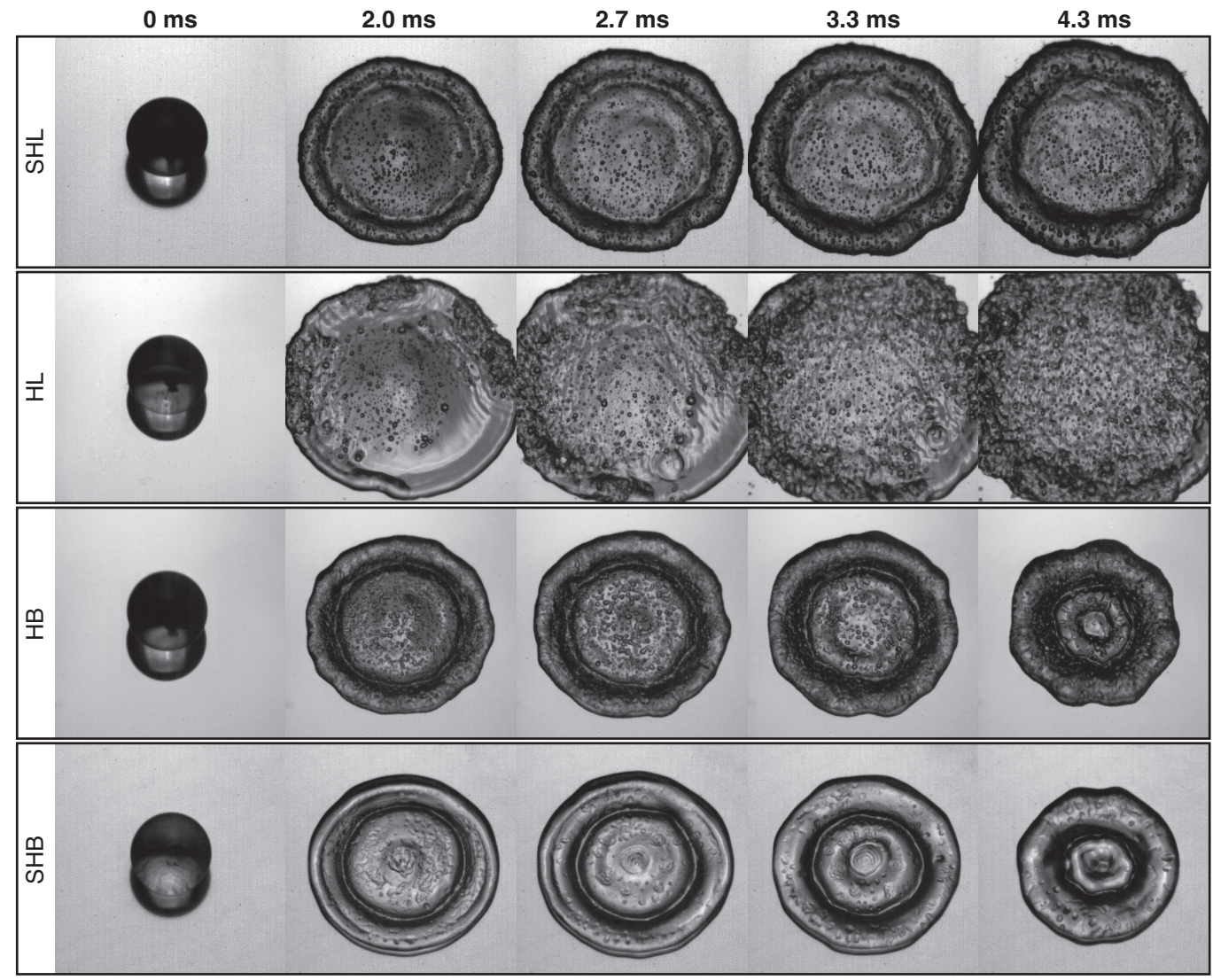

Figure 4: Top view of droplet spreading at $T_{s}=150^{\circ} \mathrm{C}$ and $W e=40$ on all four surfaces. These images show the existence of vapor bubbles that form during impingement at the solid-liquid interface on the HB and SHB surfaces, as well as the HL and SHL surfaces. 
a hydrophobic surface) $[33,34,35]$. Thus, bubble growth structure on the hydrophobic surface facilitates the formation of a vapor blanket, which leads to transition to film boiling at lower temperatures. A schematic drawing of the expected bubble growth is shown in Fig. 6. Early transition to film boiling has also been observed for hydrophobic pool boiling [36] and immersed hydrophobic solid spheres [37].

To evaluate LFP dependence on Weber number, the experiments were repeated at the same surface temperature $\left(T_{s}=275^{\circ} \mathrm{C}\right)$ as the top panel of Fig. 5 but at a higher $W e(W e=100)$. This is shown in the bottom panel of Fig. 5. The images depict that atomization occurs on the HL and SHL surfaces, as it did for the lower Weber number case, though both exhibit more violent atomization, as well as peripheral droplet break up associated with the increased kinetic energy at impact. Furthermore, the rebound on the SHL surface is characterized by the formation of a vertical jet, which has only been observed on textured hydrophilic surfaces and may be associated with "converging liquid motion toward the axis of the flattened drop" [13]. Interestingly, the droplet on the HB surface does atomize at this $W e$ indicating that the LFP at this Weber number must occur at a higher temperature. This result suggests that LFP increases with Weber number on the HB surface, a trend that is consistent with hydrophilic surfaces $[10,12,13]$. This trend occurs as a result of larger inertial impacts requiring higher vapor production at the interface to maintain a stable vapor film layer.

Remarkably, Fig. 5 shows that atomization is absent on the SHB surface at $T_{s}=275^{\circ} \mathrm{C}$ for both $W e$. As will be shown in the following section, atomization does not occur on the SHB surface at this surface temperature for any of the $W e$ explored. To investigate atomization behavior on the SHB surface further, experiments were conducted at other surface temperatures and various Weber numbers. We found that atomization was generally minimal and only present for a few specific scenarios. As an example, two impingement events on the SHB surface are shown in Fig. 7, which are representative of scenarios with the largest amount of atomization observed on the SHB surface over the entire regime explored. The top row represents SHB impingement at $W e=50$ and $T_{s}=175^{\circ} \mathrm{C}$ while the second and third row correspond to $W e=100$ and $T_{s}=220^{\circ} \mathrm{C}$ for the SHB and HL surfaces, respectively. Impingement on the HL surface is shown for visual comparison. As is evident in these images, the amount of atomization that occurs on the superhydrophobic surface is almost indistinguishable, in accordance with a previous study of sessile droplets on heated superhydrophobic surfaces of 


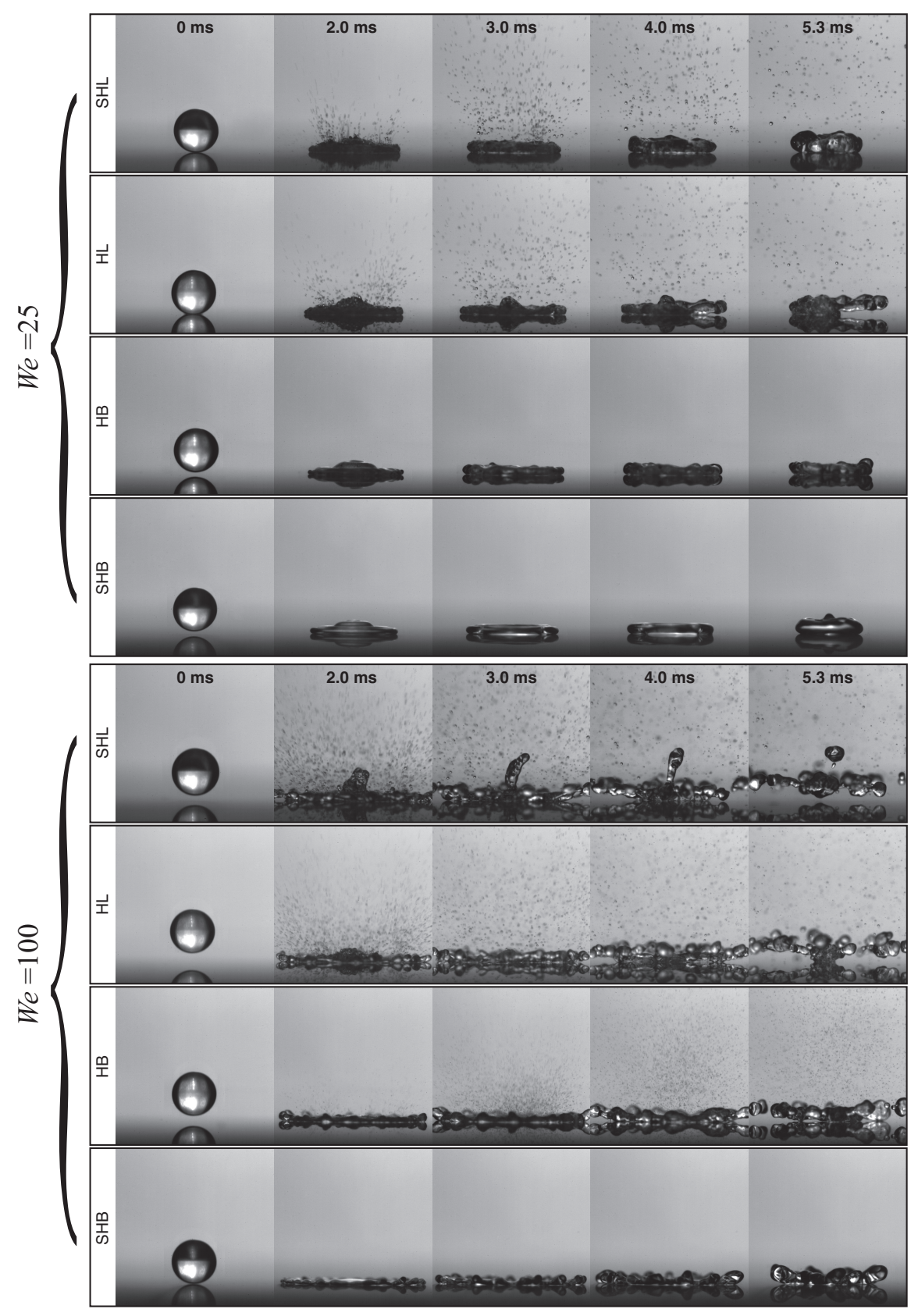

Figure 5: Droplet impingement on all four surfaces at $T_{s}=275^{\circ} \mathrm{C}$ and $W e=25$ (top) and 100 (bottom). 


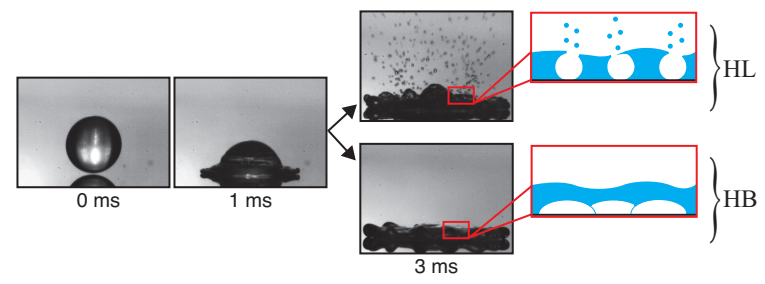

Figure 6: Schematic drawings of bubble dynamics inside the droplet during spreading on the $\mathrm{HL}$ and $\mathrm{HB}$ surfaces for $T_{s}=275^{\circ} \mathrm{C}$ and $W e=25$.

similar solid fractions [27].

Minimized atomization during contact boiling is attributed to the hydrophobic texture (micro-pillars) and rationalized as follows. First, the pillars do not permit liquid intrusion into the open space between them (even if liquid penetrates at the impingement point due to stagnation pressure, it is likely that the liquid returns to the Cassie state due to the elevated temperature of the surface [38]). The prevailing Cassie state results in a reduction of contact area between solid and liquid (solid fraction is $11 \%$ for the current case) and thus heat transfer to the liquid is decreased. Second and more importantly, the cavities in between the micro-pillars allow vapor to continuously escape from underneath the droplet, thereby mitigating vapor bubble formation and atomization. This is drastically different from behavior on other surface types during contact boiling where vapor must vent through the droplet itself, causing atomization.

To further investigate this idea, impingement events were recorded from a top view on all four surfaces in the contact boiling regime as shown in Fig. 8 at $W e=50$ and $T_{s}=200^{\circ} \mathrm{C}$. These photographs depict boiling activity on the SHL, HL and HB surfaces, which can be easily recognized by the opaque nature of the droplet. In contrast, the droplet on the SHB surface does not display boiling and remains transparent suggesting that vapor transport from underneath suppresses bubble activity. Thus, droplet impingement on the superheated SHB surface is tantamount to what occurs on the other surfaces (SHL, HL and HB) in the film boiling regime, namely, vapor is able to freely escape from underneath the droplet through the vapor film. This alternate flow path for vapor flux minimizes vapor bubbles forming within the droplet on these surfaces, thus suppressing atomization. The open space in the hydrophobic pillar forest allows vapor to escape in a similar manner even when contact prevails. One may thus analogously refer to droplet impingement 


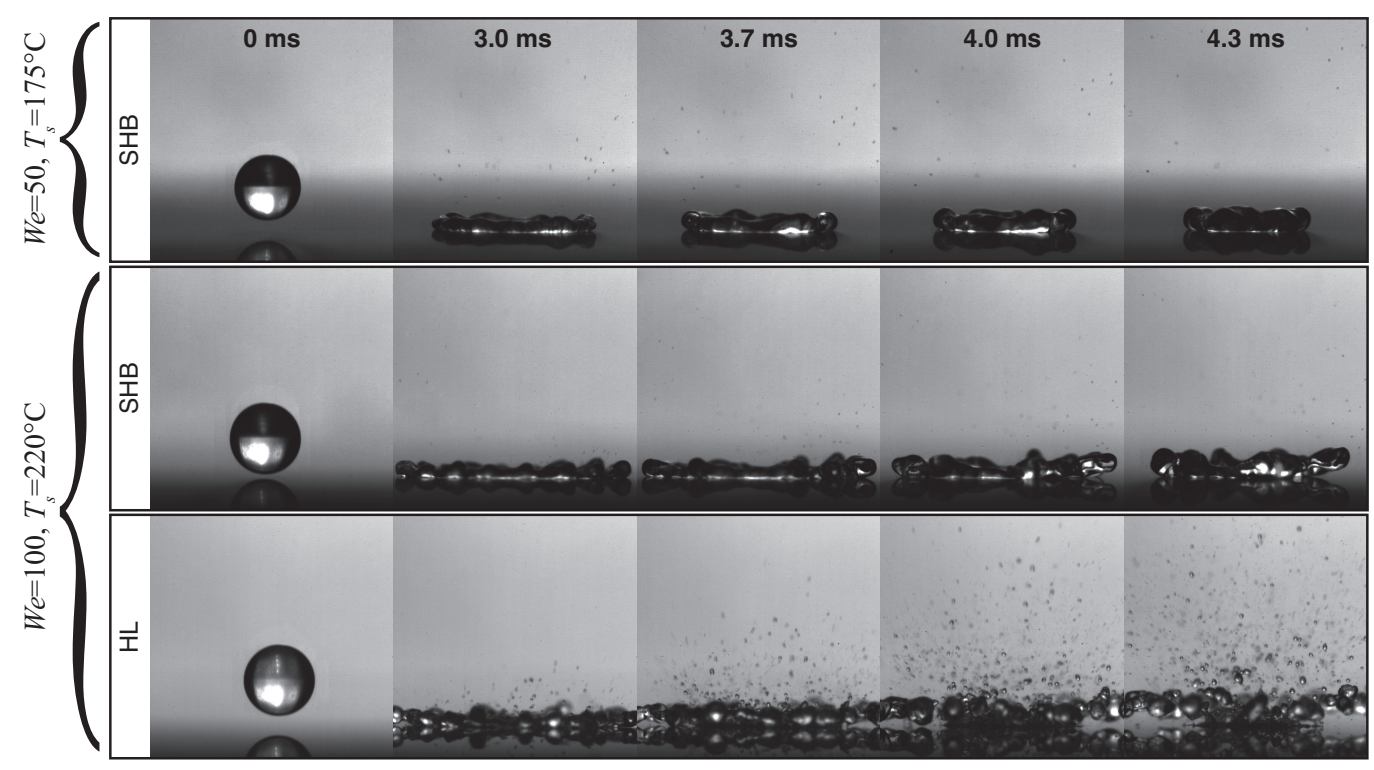

Figure 7: droplet impingement on the SHB surface at $T_{s}=175^{\circ} \mathrm{C}$ and $W e=50$ (top panel) and $220^{\circ} \mathrm{C}$ and $W e=100$ (middle panel). Both of these scenarios represent cases where the most atomization was observed on the SHB surface. Dynamics on the HL surface are shown in the bottom panel for comparison at the second set of conditions. 


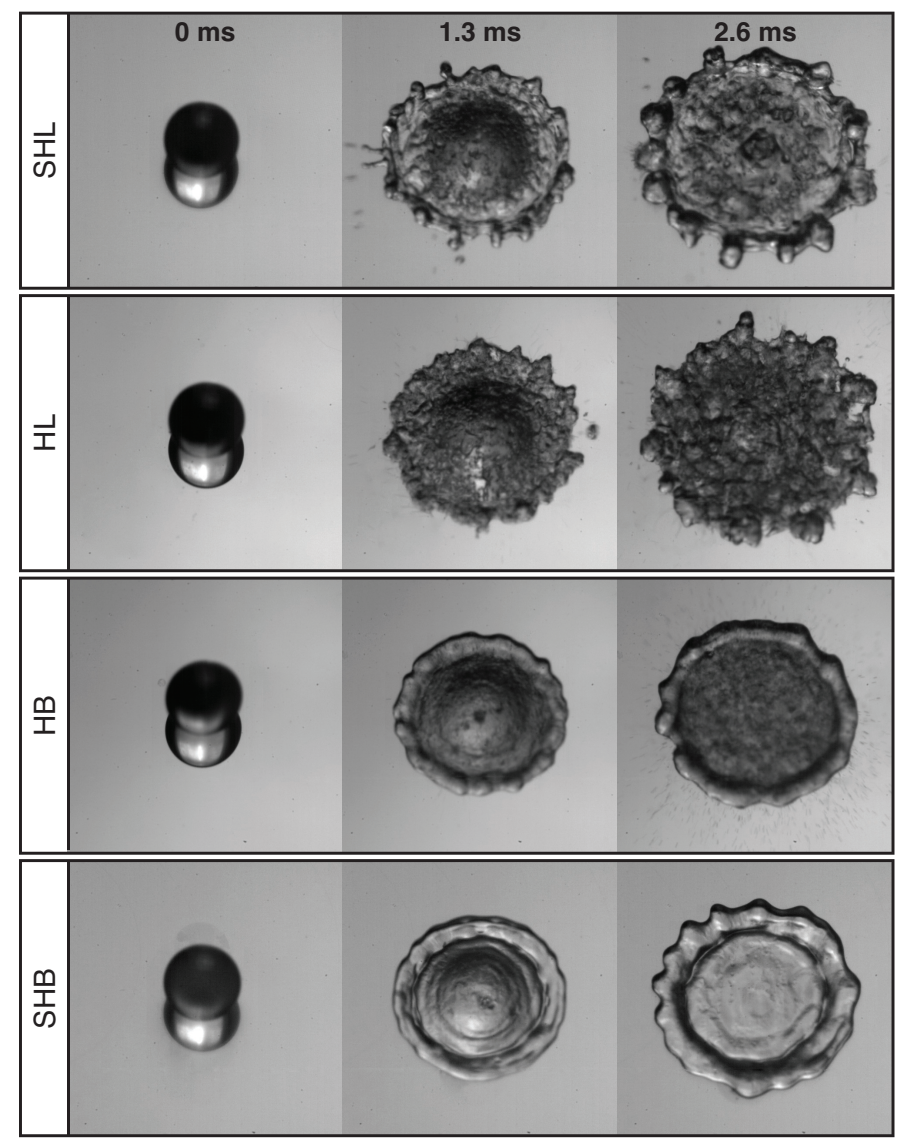

Figure 8: Top view of the impingement event for $W e=50$ and $T_{s}=200^{\circ} \mathrm{C}$ on all four surfaces to demonstrate minimal boiling behavior on SHB surface made evident by the transparency of the spreading droplet.

on the SHB surface in the contact boiling regime as a pseudo-Leidenfrost effect. As discussed earlier, no atomization was observed on the SHB surface at $T_{s}=275^{\circ} \mathrm{C}$ at any $W e$ indicating that a stable film boiling regime exists for the SHB surface and this will be discussed in the next section.

But if vapor is free to escape through the superhydrophobic interface during contact boiling, why does atomization on the SHB surface occur at all? We hypothesize that if vapor production at the solid-liquid interface is higher than the rate at which vapor can escape through the textured interface, vapor will build up in the liquid with a force proportional to the rate of vapor generation [5] forming "domes" (see Fig. 4). These domes, which can 
burst in a similar way to bubbles formed on a different surface type, are the cause of the minimally observed atomization but only induces atomization when the droplet becomes sufficiently thin during spreading/retraction (i.e. for high enough Weber numbers; discussed in next section). Future work should explore different types of superhydrophobic surfaces to quantify the relationship between resistance to vapor flow through the texture and the corresponding magnitude of atomization.

\subsection{Atomization Regime Maps}

Atomization regime maps were constructed for all surfaces over the range of $10<W e<225$ and $150^{\circ} \mathrm{C}<T_{s}<415^{\circ} \mathrm{C}$ as shown in Fig. 9. Circular symbols (accompanied by shaded regions) represent scenarios where atomization did occur, while triangular ones represent scenarios where atomization did not, where transitional boundaries fall in between. Each scenario was repeated 5 to 12 times with more repetitions near the boundaries. We begin by describing behavior on the HL and SHL surfaces and note that atomization always occurred for all temperatures between $125^{\circ} \mathrm{C}$ and the LFP regardless of the $W e$. Transition to film boiling on these surfaces increases with $W e$, which agrees with what has been previously reported $[10,12,13]$. This is due to an increase in inertial pressure at impact associated with increasing $W e$, which requires a higher surface temperature to maintain the vapor blanket at the interface, as stated earlier.

Tran et al. showed that atomization resumes on smooth hydrophilic surfaces at surface temperatures approaching $500^{\circ} \mathrm{C}$, and called this regime "spraying film boiling" [12]. In the cases considered here, atomization was never observed above the LFP on any of the surfaces, indicating that the spraying film boiling regime was likely not reached in the present experiments. Results also show that the LFP is lower for the SHL surface than for the HL one for all $W e$. This occurs because surface texturing effectively increases the solid surface area, which increases heat transfer to the droplet, promoting the formation of a stable vapor layer at lower surface temperature [13].

Next, we discuss the atomization regime map for the HB surface (third panel in Fig. 9). The map is similar in form to the HL and SHL maps, but shows two exceptions. First, no atomization occurs at $W e \approx 10$ for any $T_{s}$, or at $T_{s} \approx 125^{\circ} \mathrm{C}$ for any $W e$. The former observation is attributed to minimal spreading on the hydrophobic surface at relatively low $W e$, which results in 

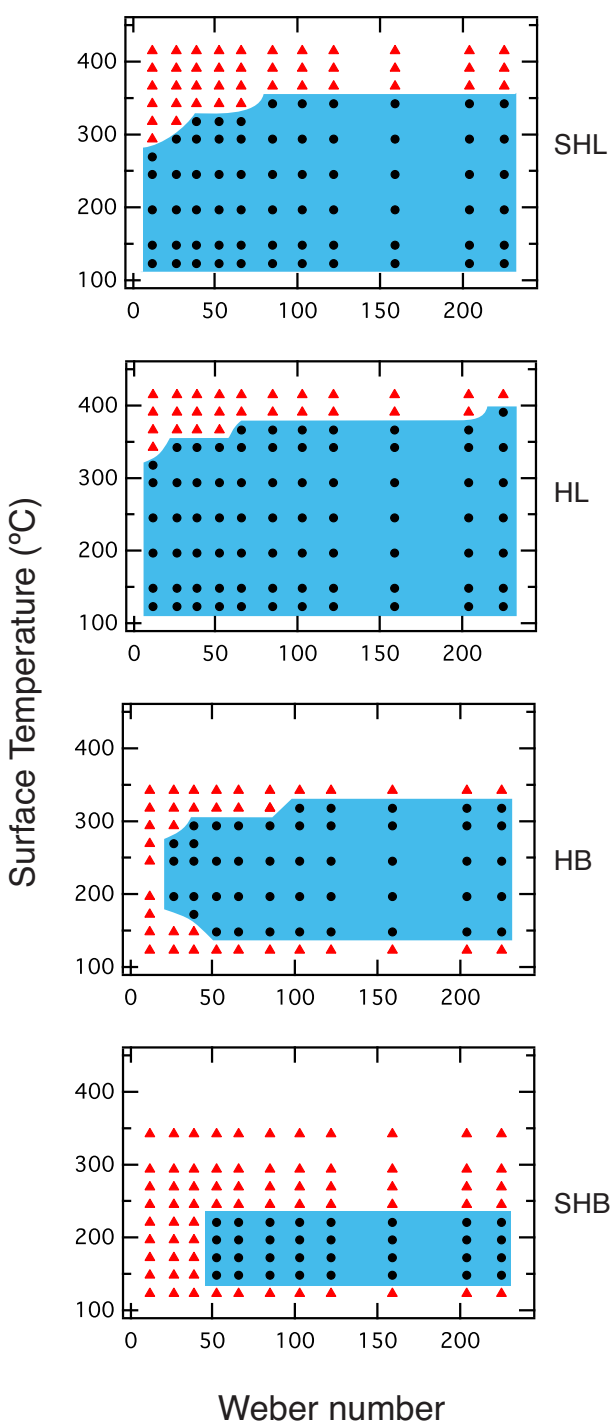

Figure 9: Atomization regime map for all four surfaces. Circles represent scenarios where atomization occurred, while triangles represent scenarios where atomization was not observed. 
a thicker spreading drop. Thus, while vapor bubbles still form at the solidliquid interface, they never penetrate the free surface of the droplet. The latter is due to the relatively low heat transfer rate into the droplet. Here, the short time the droplet spends flat on the surface is much less than the time it takes for atomization to begin on the hydrophilic surface $(\sim 50 \mathrm{~ms})$. The second difference regarding impingement on the HB surface is the lower surface temperature associated with transition to film boiling relative to both the HL and SHL surfaces for all $W e$ explored. A hypothesis for this behavior was formulated and described in conjunction with Fig. 5, and is related to the attraction of vapor bubbles to the surface.

Atomization behavior differs dramatically on the SHB surface. First, the map (bottom panel of Fig. 9) depicts a significantly smaller atomization or contact boiling regime than the other three surfaces. Furthermore, the intensity of the atomization in this regime is significantly less than that on other surfaces as noted previously (see Fig. 7). This was attributed to the ability of vapor to escape through the cavities in the pillar forest, which, being similar to vapor transport on other surface types at the LFP, was named the pseudo-Leidenfrost state. This map shows that a transition exists $\left(T_{s} \approx\right.$ $245^{\circ} \mathrm{C}$ ) from this pseudo-Leidenfrost state to a more stable Leidenfrost state, where no atomization was observed at all. This transition indicates that increasing surface temperature (thus increasing vapor production) stimulates more vapor production such that the vapor layer is now taller than the pillars, which yields a larger escape route for vapor.

Figure 10 shows the LFP (based on the regime maps in Fig. 9) as a function of $W e$. The top panel includes LFP values for the SHL and HL surfaces, as well as data from Tran et al. [12, 13] and Bertola [10]. The bottom panel shows the LFP for all surfaces in this work. Error bars are based on the resolution of temperature ranges at which experiments were performed, $25^{\circ} \mathrm{C}$. Data from Tran et al. represent LFP values for droplet impingement on heated smooth hydrophilic $[+]$ and two types of textured hydrophilic silicon wafers similar to the ones used in this work. The pillar diameter on their textured hydrophilic surfaces was $9 \mu \mathrm{m}$ and the pillar height was $8 \mu \mathrm{m}$, while the pitch values were $29 \mu \mathrm{m}[\triangle]$ and $13 \mu \mathrm{m}[\nabla]$. Surface temperature values reported by Tran et al. in their publication $[12,13]$ were based on thermocouple readings embedded in the stainless steel holder upon which the wafers were placed rather than actual wafer surface temperatures. These values were later corrected by Bertola to represent actual surface temperature and the corrected values are the ones shown here. The second set of 
data are from Bertola [ $\square]$ and represent experiments performed on a heated aluminum surface with average surface roughness of $0.1 \mu \mathrm{m}$. All trends in the top panel of Fig. 10 show that the LFP increases with $W e$. LFP values on the hydrophilic surface show very good agreement with results of Tran et al. for $W e<50$, but exhibit an offset of about $20^{\circ} \mathrm{C}$ at higher Weber numbers, which falls within the resolution of the experiments. Superhydrophilic LFP values reported in this work differ from those of Tran et al. by $50^{\circ} \mathrm{C}$ and $75^{\circ} \mathrm{C}$ at low and high Weber numbers, respectively. This is rather unexpected since their texture also consisted of micro-pillars, but differences might be related to pillar diameter where those used by Tran et al. were $50 \%$ larger than the ones used here. This would further explain their lower LFP values which are a consequence of higher heat transfer rates. Results from Bertola match more closely our data for the SHL surfaces. This may be due to roughness on the aluminum surface, which approximates the superhydrophilic scenario, although some discrepancy is expected due to the different material used. Data in the bottom panel of Fig. 10 shows that the LFP on the SHL surface is nominally $12 \%$ less than that on the HL surface over the entire We range explored, while the LFP on the HB and SHB surfaces are respectively $20 \%$ and $40 \%$ smaller than for the HL surface. Moreover, the SHB surface exhibited no dependence of the LFP on the Weber number over the entire $W e$ range explored. Given the probabilistic nature of the transitional boundaries aforementioned, it is difficult to determine this dependence definitively.

\subsection{Maximum Spread Diameters}

In this section, we discuss the relationship between the normalized maximum spread diameter $\left(D_{\max } / D\right)$ at the LFP and the Weber number as shown in Fig. 11. Weber numbers of 100 and greater generally resulted in satellite droplet ejection (matching the work of Bertola [10]) thus limiting the $W e$ range that was explored. Maximum spread diameters on a superhydrophobic surface at room temperature are typically provided for comparison when discussing Leidenfrost droplet rebound since both undergo a repelling interaction $[39,40]$. We do likewise here and thus the top panel depicts our results for impingement on the SHB surface at room temperature alongside with results for the HL and SHL surfaces at their respective LFP values (where the LFP at a given $W e$ was defined in Sec. 3.2). Also shown are experimental data from Antonini et al. (superhydrophobic surface at room temperature) [24] and Tran et al. (hydrophilic and superhydrophilic surfaces at their respective LFP values) [13] for comparison. 


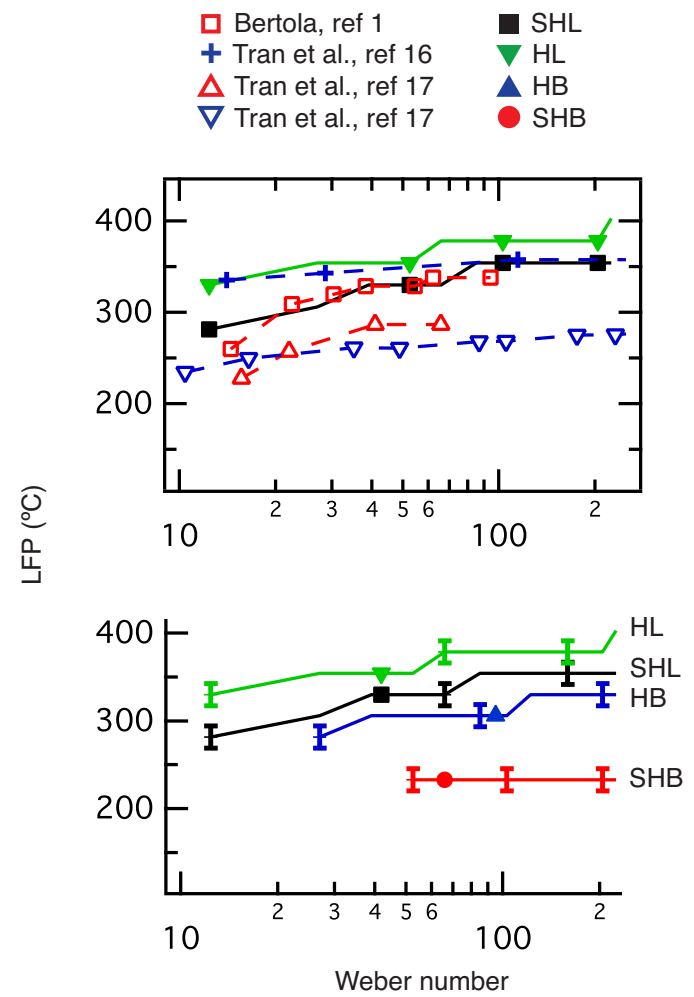

Figure 10: Transition temperature to the Leidenfrost point or LFP as a function of $W e$. Top panel: Present results shown with data from Bertola [10] and Tran et al. [12]. Bottom panel: Present results for all four surfaces. 


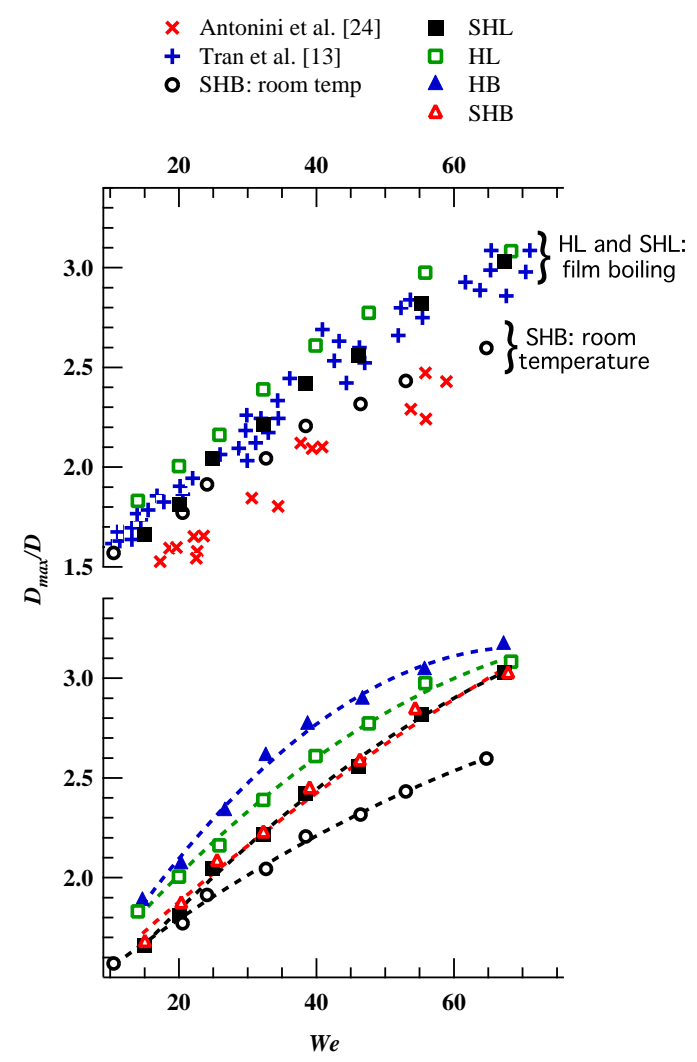

Figure 11: Normalized maximum spread diameter as a function of $W e$ at the corresponding LFP values for each surface and SHB at room temperature. Top panel: Present results shown with data from Antonini et al. [24] and Tran et al. [13]. Bottom panel: Present results for all four surfaces with a curve fit for clarity. 
Our data compares well with the two data sets from the literature in Fig. 11. All data sets show that $D_{\max } / D$ increases with increasing $W e$ for all scenarios, as expected. However, the maximum diameter is lower on the superhydrophobic surface at room temperature than at the LFP for the hydrophilic and superhydrophilic surfaces. This should not be surprising given two facts. First, even though the vapor blanket "insulates" the droplet from the heated surface on a Leidenfrost impingement, the temperature of the bottom of the droplet is still close to saturation temperature since it is continuously vaporizing. Because the bottom of the droplet houses the largest velocity gradients [41], the reduction of liquid viscosity (due to increased temperature) is significant and allows for larger spreading. Second, Leidenfrost spreading encounters virtually no solid-liquid contact and thus approaches an infinite slip scenario [42], which is in contrast with spreading on the SHB surface at room temperature, which experiences no shear over cavities but frictional resistance over the pillars.

Having shown agreement between our data and that of previous researchers, focus is now turned to results for all surfaces considered in this work. The bottom panel of Fig. 11 constitutes our results shown in the top panel with the addition of the HB and SHB surface results at their respective LFP values. A curve fit to each data set is shown for clarity. Since no transition to Leidenfrost behavior on the SHB surface occurred for $W e<50$ (atomization was absent at all temperatures), impingement was evaluated at a constant surface temperature of $230^{\circ} \mathrm{C}$, the $\mathrm{LFP}$ value at higher $W e$. The data here shows that the maximum spread diameter is larger on the HB and SHB surface at their respective LFPs for all $W e$ than for the SHB surface at room temperature, as was the case with the HL and SHL surfaces. More interestingly, the figure shows that $D_{\max } / D$ is lower for both textured surfaces (SHL and SHB), which virtually overlap each other, than for the smooth surfaces (HL and HB) for all We. This difference is greater than the uncertainty of the experiments (which is not depicted on the figure as it is never greater than the scale of the markers), which is low given that length calibration uncertainty does not play a role due to the manner of normalization. For example, at $W e \approx 33, D_{\max } / D=2.61$ on the HB surface, but only 2.22 on the SHB and SHL surfaces, yielding an $18 \%$ increase, while uncertainty was about $2 \%$ on either surface. The differences in maximum spread diameter across all the surfaces suggest that Leidenfrost rebound is not independent of surface type. At first glance, it seems reasonable to assume that the considerable differences in LFP temperatures across the different surfaces may 
cause the differences in maximum diameters. However, we see that $D_{\max } / D$ on the SHL and SHB surfaces collapse to nearly the same curve even though the LFP on the former is generally $100^{\circ} \mathrm{C}$ greater than on the latter. This suggests that surface architecture plays a dominant role on determining the maximum diameter over the temperature at which LFP occurs.

Equivalent maximum spreading diameters on both textured surfaces indicate that a vapor layer prevails at these interfaces during spreading. This follows because significant contact between liquid and solid would exhibit drastically different wetting dynamics between the surfaces. On the SHL surface, liquid-solid contact would cause the liquid to adhere to the pillars (due to wicking) in the manner that was discussed by Kwon et al. [5], while significantly less adhesion would occur on the SHB texture. Given these differential wetting preferences, maximum spreading diameters on the SHL surface would be smaller than on the SHB surface, which is not the case. On the other hand, the relative decrease in spreading diameters between the textured surfaces and the smooth ones can then be attributed to the differences in vapor flow under the droplet. Vapor flow through a forest of pillars experiences significantly higher resistance than over a smooth surface. This difference results in larger spreading diameters on the smooth surfaces relative to the textured ones.

Differences in maximum spread diameters between the HB and HL surface are now discussed. While this difference is not as large as that of the HB and textured surfaces, it is a measurable difference nonetheless. This is interesting because both surfaces are smooth and hence diameter discrepancies cannot be attributed to the textural differences. Once again, we highlight that farther spreading on the HB surface due to decreased viscosity is unlikely since surface temperature is lower on HB surface at LFP than on the HL surface. Bernardin and Mudawar performed two seminal works which brought much insight to the formation of the Leidenfrost vapor layer during droplet impingement $[44,45]$. They developed a model which shows that at the LFP temperature, "contact between liquid and solid can exist for a relatively short duration" [45]. This contact is brief but necessary to continue to replenish vapor at the interface. Thus, we might expect two effects responsible for smaller spreading diameters on the HL surface relative to the HB surface. First, stronger attraction during these brief occurrences is expected on the HL surface. In the case of the textured surfaces, this effect might be obscured by the dominant effect of the friction posed by vapor flow around the pillars. Second, bubble formation on the HL surface may 
impose an adverse hydrodynamical condition on the spreading liquid. This idea is concomitant with protruding menisci increasing friction rather than providing a favorable slip condition at the solid-liquid interface [46].

\section{Conclusion}

In this paper, the hydrodynamics of a single droplet impinging on a superheated solid surface were analyzed. Previous research has focused on smooth and textured hydrophilic surfaces and, as such, experiments here were performed on a smooth hydrophobic and a micro-pillar textured superhydrophobic surface. A smooth hydrophilic and a micro-pillar textured superhydrophilic surface were also used for comparison, as well as data available in the literature. The ranges of surface temperatures and Weber numbers considered were $150^{\circ} \mathrm{C}$ to $415^{\circ} \mathrm{C}$ and 10 to 225 , respectively. High-speed images were acquired and presented to qualitatively discuss the associated impingement dynamics across the different surface types. Results were quantified into regime maps which depict where atomization is present. Finally, the maximum spread diameter at the LFP prior to peripheral droplet breakup for each surface was quantified and compared across all surfaces as a function of the Weber number. Results can be summarized as follows for impingement on the hydrophobic surface explored here:

- Atomization is absent at low Weber numbers ( $W e \approx 10$ ) for all surface temperatures and at low surface temperatures $\left(T_{s} \approx 125^{\circ} \mathrm{C}\right)$ for all Weber numbers. The former occurs because the droplet does not spread as far as it does on a hydrophilic surface prior to retraction resulting in a thicker spreading film, while the latter because the droplet spends less time in contact with the surface. Both of these behaviors prevent vapor bubbles from bursting.

- Transition to the Leidenfrost regime occurs at lower surface temperatures than on the hydrophilic or superhydrophilic surfaces for all Weber numbers explored. Given the attraction of vapor bubbles to the hydrophobic surface, a vapor blanket at the solid-liquid interface is more readily formed.

- At the Leidenfrost point, the droplet spreads out farther prior to retraction compared to all other surfaces at their respective LFP. This behavior is not attributed to lower viscosity since the LFP values of some of the other surfaces are higher. 
For impingement on the superhydrophobic surface:

- Atomization is significantly less intense than on all other surface types and occurs for a much narrower range of parameters. The hydrophobic micro-pillars at the interface cause water to be suspended above them and thus vapor escapes from underneath the droplet, which promotes Leidenfrost-like behavior at all conditions.

- Transition to the Leidenfrost regime occurs at a much lower surface temperature than all other surface types.

- Maximum spread diameter at the LFP is lower than both the hydrophobic and hydrophilic surfaces for all $W e$. This indicates that some solidliquid contact may prevail during spreading, thus exerting frictional resistance on the flow.

\section{Acknowledgements}

This material is based upon work supported by the National Science Foundation under Grant No. 1066356.

[1] J. Li, Y. Hou, Y. Liu, C. Hao, M. Li, M. K. Chaudhury, S. Yao, and Z. Wang, "Directional transport of high-temperature janus droplets mediated by structural topography," Nature Physics, vol. advance online publication.

[2] D. A. del Cerro, A. G. Marin, G. R. B. E. Romer, B. Pathiraj, D. Lohse, and A. J. Huis in 't Veld, "Leidenfrost point reduction on micropatterned metallic surfaces," Langmuir, vol. 28, no. 42, pp. 15106-15110, 2012. PMID: 23020737.

[3] H. Fujimoto, S. Watanabe, T. Okamoto, T. Hama, and H. Takuda, "Photographic study of hydrodynamics of drops of aqueous polymer solution impinging on hot solid," Experimental Thermal and Fluid Science, vol. 60 , pp. $66-74,2015$.

[4] M. Gradeck, N. Seiler, P. Ruyer, and D. Maillet, "Heat transfer for leidenfrost drops bouncing onto a hot surface," Experimental Thermal and Fluid Science, vol. 47, pp. 14 - 25, 2013. 
[5] H.-m. Kwon, J. C. Bird, and K. K. Varanasi, "Increasing leidenfrost point using micro-nano hierarchical surface structures," Applied Physics Letters, vol. 103, no. 20, pp. 201601, 2013.

[6] C. Lee, D. Kim, H. Kim, and K. Kim, "Dynamic behavior and microexplosion characteristics of impinging droplets on a high-temperature surface," Journal of Visualization, vol. 18, no. 1, pp. 59-70, 2015.

[7] H. Kim, B. Truong, J. Buongiorno, and L.-W. Hu, "On the effect of surface roughness height, wettability, and nanoporosity on leidenfrost phenomena," Applied Physics Letters, vol. 98, no. 8, pp. 083121, 2011.

[8] H. Fujimoto, Y. Oku, T. Ogihara, and H. Takuda, "Hydrodynamics and boiling phenomena of water droplets impinging on hot solid," International Journal of Multiphase Flow, vol. 36, no. 8, pp. 620 - 642, 2010.

[9] X. Li, X. Ma, and Z. Lan, "Behavioral patterns of drop impingement onto rigid substrates with a wide range of wettability and different surface temperatures," AIChE Journal, vol. 55, no. 8, pp. 1983-1992, 2009.

[10] V. Bertola, "An impact regime map for water drops impacting on heated surfaces," International Journal of Heat and Mass Transfer, vol. 85, pp. $430-437,2015$.

[11] J. D. Bernardin, C. J. Stebbins, and I. Mudawar, "Mapping of impact and heat transfer regimes of water drops impinging on a polished surface," International Journal of Heat and Mass Transfer, vol. 40, no. 2, pp. $247-267,1997$.

[12] T. Tran, H. J. J. Staat, A. Prosperetti, C. Sun, and D. Lohse, "Drop impact on superheated surfaces," Phys. Rev. Lett., vol. 108, pp. 036101, Jan 2012.

[13] T. Tran, H. J. J. Staat, A. Susarrey-Arce, T. C. Foertsch, A. van Houselt, H. J. G. E. Gardeniers, A. Prosperetti, D. Lohse, and C. Sun, "Droplet impact on superheated micro-structured surfaces," Soft Matter, vol. 9, pp. 3272, 2013.

[14] O. Engel, "Waterdrop collisions with solid surfaces," Journal of Research of the National Bureau of Standards, vol. 54, pp. 281-298, 1955. 
[15] L. Wachters and N. Westerling, "The heat transfer from a hot wall to impinging water drops in the spherical state," Chemical Engineering Science, vol. 21, pp. 1047-1056, 1966.

[16] J. G. Leidenfrost, De aquae communis nonnullis qualitatibus tractatus. Ovenius, 1756.

[17] S. Chandra and C. Avedisian, "Observations of droplet impingement on a ceramic porous surface," International Journal of Heat and Mass Transfer, vol. 35, no. 10, pp. 2377 - 2388, 1992.

[18] W. Zhang, T. Yu, J. Fan, W. Sun and Z. Cao, "Droplet impact behavior on heated micro-patterned surfaces," Journal of Applied Physics, vol. 119, pp. 114901, 2016.

[19] J. Padilla and V. P. Carey, "Water droplet vaporization on superhydrophilic nanostructured surfaces at high and low superheat," Proceedings of the ASME 2014 International Mechanical Engineering Congress and Exposition, v. 8B, pp. V08BT10A070, 2014.

[20] E.-S. R. Negeed, S. Hidaka, M. Kohno, and Y. Takata, "High speed camera investigation of the impingement of single water droplets on oxidized high temperature surfaces," International Journal of Thermal Sciences, vol. 63, pp. 1 - 14, 2013.

[21] H. Nair, H. J. J. Staat, T. Tran, A. van Houselt, A. Prosperetti, D. Lohse, and C. Sun, "The Leidenfrost temperature increase for impacting droplets on carbon-nanofiber surfaces," Soft Matter, vol. 10, pp. 2102-2109, 2014.

[22] A. Moita and A. Moreira, "Scaling the effects of surface topography in the secondary atomization resulting from droplet/wall interactions," Experiments in Fluids, vol. 52, no. 3, pp. 679-695, 2012.

[23] A. V. Mahulkar, G. B. Marin, and G. J. Heynderickx, "Droplet-wall interaction upon impingement of heavy hydrocarbon droplets on a heated wall," Chemical Engineering Science, vol. 130, pp. 275 - 289, 2015.

[24] C. Antonini, I. Bernagozzi, S. Jung, D. Poulikakos, and M. Marengo, "Water drops dancing on ice: How sublimation leads to drop rebound," Physical Review Letters, vol. 111, pp. 014501, 2013. 
[25] S. H. Kim, J. Y. Kang, H. S. Ahn, H. J. Jo, and M. H. Kim, "Study of Leidenfrost mechanism in droplet impacting on hydrophilic and hydrophobic surfaces," International Journal Air-Conditioning and Refrigeration, vol. 21, no. 4, pp. 1350028, 2013.

[26] J. Yong Park, A. Gardner, W. P. King, and D. G. Cahill, "Droplet impingement and vapor layer formation on hot hydrophobic surfaces," Journal of Heat Transfer, vol. 136, pp. 092902, 2014.

[27] R. Hays, D. Maynes, and J. Crockett, "Thermal transport to droplets on heated superhydrophobic substrates," International Journal of Heat and Mass Transfer, vol. 98, pp. 70 - 80, 2016.

[28] C. Kruse, T. Anderson, C. Wilson, C. Zuhlke, D. Alexander, G. Gogos and S. Ndao, "Extraordinary shifts of the Leidenfrost temperature from multiscale micro/nanostructured surfaces," Langmuir, vol. 29, pp. 9798 $-9806,2013$.

[29] Y. Takata, S. Hidaka, J. M. Cao, T. Nakamura, H. Yamamoto, M. Masuda and T. Ito, "Effect of surface wettability on boiling and evaporation," Energy, vol. 30, pp. 209 -220, 2005.

[30] H. Kim, B. Truong, J. Buongiorno, and L. Hu, "On the effect of surface roughness height, wettability, and nanoporosity on Leidenfrost phenomena," Applied Physics Letters, vol. 98, pp. 083121, 2011.

[31] R. Wenzel, "Resistance of solid surfaces to wetting by water," Industrial Engineering Chemistry., vol. 28, no. 8, pp. 988-994, 1936.

[32] A. Cassie and S. Baxter, "Wettability of porous surfaces," Trans Faraday Soc., vol. 40, pp. 546-551, 1944.

[33] Y. Nam, J. Wu, G. Warrier, and Y. S. Ju, "Experimental and numerical study of single bubble dynamics on a hydrophobic surface," Journal of Heat Transfer, vol. 131, pp. 121004, 2009.

[34] H. Jo, H. S. Ahn, S. Kang, and M. H. Kim, "A study of nucleate boiling heat transfer on hydrophilic, hydrophobic and heterogeneous wetting surfaces," International Journal of Heat and Mass Transfer, vol. 54, no. 25?26, pp. $5643-5652,2011$. 
[35] H. Jo, H. S. Park, and M. H. Kim, "Single bubble dynamics on hydrophobic?hydrophilic mixed surfaces," International Journal of Heat and Mass Transfer, vol. 93, pp. 554 - 565, 2016.

[36] L.-W. Fan, J.-Q. Li, D.-Y. Li, L. Zhang, and Z.-T. Yu, "Regulated transient pool boiling of water during quenching on nanostructured surfaces with modified wettability from superhydrophilic to superhydrophobic," International Journal of Heat and Mass Transfer, vol. 76, pp. 81 - 89, 2014.

[37] I. U. Vakarelski, N. A. Patankar, J. O. Marston, D. Y. C. Chan, and S. T. Thoroddsen, "Stabilization of leidenfrost vapour layer by textured superhydrophobic surfaces," Nature, vol. 489, pp. 274-277, 2012.

[38] C. Clavijo, J. Crockett, and D. Maynes, "Wenzel to cassie transition during droplet impingement on a superhydrophobic surface," Submitted, 2016 .

[39] A.-L. Biance, F. Chevy, C. Clanet, G. Lagubeau, and D. Quéré, "On the elasticity of an inertial liquid shock," Journal of Fluid Mechanics, vol. 554, pp. 47-66, 2006.

[40] M. Reyssat, D. Richard, C. Clanet, and D. Quéré, "Dynamical superhydrophobicity," Faraday Discuss., vol. 146, pp. 19-33, 2010.

[41] G. Rosengarten, A. Tetuko, K. Li, A. Wu, and R. Lamb, "The effect of nano-structured surfaces on droplet impingement heat transfer," Proceedings of the ASME 2011 International Mechanical Engineering Congress and Exposition, v. 6, pp. 1029-1036, 2011.

[42] C. E. Clavijo, J. Crockett, and D. Maynes, "Effects of isotropic and anisotropic slip on droplet impingement on a superhydrophobic surface," Physics of Fluids, vol. 27, no. 12, pp. 122104, 2015.

[43] C. Ishino, M. Reyssat, E. Reyssat, K. Okumura, and D. Quéré, "Wicking within forests of micropillars," Europhysics Letters, vol. 79, pp. 56005, 2007.

[44] J. D. Bernardin, and I. Mudawar, "The Leidenfrost Point: Experimental Study and Assessment of Existing Models," Journal of Heat Transfer, vol. 121, pp. 894-903, 1999. 
[45] J. D. Bernardin, and I. Mudawar, "A Cavity Activation and Bubble Growth Model of the Leidenfrost Point," Journal of Heat Transfer, vol. 124, pp. 864-874, 2002.

[46] A. Steinberger, C. Cottin-Bizonne, P. Kleimann, and E. Charlaix, "high friction on a bubble mattress," Nature, vol. 6, pp. 665-668, 2007. 